\title{
Pratique OBI: Um recurso de apoio a treinos para a modalidade Iniciação da Olimpíada Brasileira de Informática
}

\author{
Ana Luísa V. Solórzano ${ }^{1}$, Cassiano A. Schneider ${ }^{1}$, Andrea S. Charão ${ }^{2}$ \\ ${ }^{1}$ Curso de Ciência da Computação \\ ${ }^{2}$ Departamento de Linguagens e Sistemas de Computação \\ Universidade Federal de Santa Maria \\ Santa Maria, RS, Brasil \\ \{alsolorzano, cschneider, andrea\}einf.ufsm.br
}

\begin{abstract}
The Brazilian Olympiad of Informatics is an annual event to middleschool and high-school students. In the modality called "Initiation", participants have to answer logic problems without the use of a computer. One method to study is by resolving previous tests. In Brazil, some projects offer trains to participants in the Olympiads. However, choose specific questions to compose study material can be a tough task. This paper presents Pratique OBI, a web system to create personalized tests and to easily consult questions to support trains for the Initiation modality. We performed user tests with tutors of a project to train participants, and made improvements that are available for all.
\end{abstract}

Resumo. A Olimpíada Brasileira de Informática (OBI) é um evento anual voltado a alunos da educação básica. Na modalidade Iniciação, em que resolvemse problemas de lógica sem o uso de computador, um método de estudo é a resolução de provas anteriores. Em todo o Brasil, projetos oferecem treinos para alunos interessados na OBI, porém a seleção de questões específicas para compor aulas e simulados pode ser trabalhosa. Neste artigo, apresenta-se Pratique OBI, um sistema web que visa auxiliar no treino de participantes na modalidade Iniciação, através da criação de provas personalizadas e consulta simplificada a questões. O sistema passou por testes com usuários experientes em tutorar treinos para a OBI, incorporando melhorias que estão acessíveis à comunidade.

\section{Introdução}

O desenvolvimento do raciocínio lógico em crianças e jovens é de grande importância em seus processos de aprendizagem, pois esta habilidade auxilia na resolução de problemas e na compreensão de conceitos cada vez mais avançados [Piaget and Inhelder 1975]. Há muitas formas de desenvolver esta habilidade, incluindo abordagens com jogos ou no formato de competições [Dantas et al. 2013].

Uma competição que se enquadra neste contexto é a Olimpíada Brasileira de Informática (OBI), um evento anual promovido pela Sociedade Brasileira de Computação e organizado pela Unicamp, voltado a alunos da Educação Básica [de Oliveira Anido and Menderico 2007]. Com provas em duas modalidades, Iniciação e Programação, a OBI se apresenta como uma importante estratégia de fomento 
a jovens interessados em áreas de Ciência e Tecnologia, em especial na Computação, e de incentivo à permanência em cursos superiores. A modalidade Iniciação da OBI explora principalmente problemas de lógica, matemática e computação, resolvidos com lápis e papel. Atualmente, uma prova oficial desta modalidade possui de 15 a 40 questões, com dificuldades e conceitos lógicos distintos. O site oficial da OBI disponibiliza todas as provas em formato PDF com seus respectivos gabaritos, cuja disponibilidade é essencial para fins de estudo.

$\mathrm{Na}$ literatura, existem relatos de vários projetos que oferecem treinos para interessados em participar da OBI [de Barros et al. 2009, dos Santos et al. 2015]. Alguns benefícios deste apoio são: (i) desenvolver a autonomia do aluno, que será majoritariamente responsável pelos seus estudos para a prova, (ii) desenvolver a participação e o engajamento dos alunos durante os treinos presenciais, e (iii) motivar vínculos do aluno com a escola, visto que existem problemas com temas correlatos a disciplinas da grade curricular, o que pode impactar positivamente no desempenho escolar.

A utilização de questões da OBI é claramente uma importante ferramenta para práticas em lógica. Porém, para competidores e seus treinadores, a escolha manual de questões com níveis de dificuldade e classificações específicas pode ser trabalhosa, visto que as provas não estão disponíveis em um formato que facilite consultas. Além disso, é trabalhoso extrair questões para compor simulados ou materiais de estudo. Assim, competidores têm dificuldades em otimizar seus treinos, ficando suscetíveis a resolver questões repetidas ou deixar de estudar alguns tipos de questões. Esse cenário trouxe a motivação para o desenvolvimento de um sistema visando facilitar a consulta às provas e a elaboração de materiais de estudo com questões específicas das provas.

Neste artigo, apresenta-se Pratique OBI, um sistema web que disponibiliza consultas a todas as provas, gabaritos e questões da modalidade Iniciação. As questões são separadas de acordo com as provas em que se inserem e são classificadas pelos conceitos lógicos que abordam, podendo ser acessadas em um sistema de busca de fácil interação com o usuário. A partir do banco de provas, o usuário pode selecionar as questões desejadas e compor um documento personalizado para ser utilizado em treinos para a OBI, podendo salvá-lo em diferentes locais em formato editável ou não.

O restante deste artigo está organizado como segue. Na seção 2, discute-se alguns trabalhos relacionados com abordagens de apoio a treinos da OBI, além de recursos com formatos úteis a diferentes competições ou treinos/estudos. Na seção 3, apresenta-se o sistema Pratique OBI em maiores detalhes, juntamente com suas decisões de projeto e implementação. Na seção 4, descreve-se o processo de teste do sistema com usuários, juntamente com os resultados obtidos. Por fim, a seção 5 apresenta considerações finais sobre o trabalho.

\section{Trabalhos Relacionados}

A organização da OBI disponibiliza, em seu site oficial ${ }^{1}$, todas as provas da modalidade Iniciação desde 2003, catalogadas por nível e fase. Para cada prova, tem-se links para o documento em formato PDF e para o seu gabarito. Para algumas provas, há gabaritos comentados, com justificativas sobre cada alternativa. Além do acesso a provas anteriores, $o$

\footnotetext{
${ }^{1}$ https://olimpiada.ic.unicamp.br/
} 
site também sugere o livro "Jogos de Lógica" [Martins 2011] como material preparatório para as provas e disponibiliza algumas questões para resolução online.

Em projetos que oferecem atividades preparatórias para a OBI, é comum a resolução de problemas de provas anteriores [de Barros et al. 2009, de Barros and Oeiras 2010, de Almeida Machado et al. 2010, dos Santos et al. 2015]. Autores citam também a elaboração de materiais preparatórios para estudo, como apostilas e provas, e a realização de simulados iniciais com questões selecionadas de baixo grau de dificuldade, para identificar o nível de compreensão dos participantes na resolução de problemas de lógica. Nota-se, portanto, uma preocupação em ofertar uma variedade de recursos para apoiar os competidores.

Sabe-se que problemas semelhantes aos da OBI são encontrados em outras competições e provas. Por exemplo, o desafio Bebras $^{2}$ é uma competição internacional para disseminar o pensamento computacional entre jovens em idade escolar [Dagiené 2006]. Assim como a OBI, o Bebras realiza um desafio anual para diferentes níveis de escolaridade e de idade com provas de múltiplaescolha [Dagienė and Futschek 2008], porém a resolução das provas é feita no computador. Como recurso para estudo, alguns organizadores do Bebras disponibilizam um aplicativo para a resolução de problemas de provas passadas em formato de um jogo, com diferentes fases e interação textual com o jogador ${ }^{3}$. Esse tipo de recurso, em forma de aplicativo para o competidor, também é proposto na literatura como algo útil na preparação para a OBI [Trindade et al. 2017].

De forma mais ampla, considerando recursos voltados para instrutores e professores em geral, existem sistemas web para a geração de provas personalizadas. Por exemplo, o sistema FTD Digital ${ }^{4}$ disponibiliza o Gestor de Atividades, Simulados e Provas, que permite a criação de provas e de simulados utilizando questões de vestibulares e do ENEM, cadastradas pelos administradores do sistema. O GAPI ${ }^{5}$ é um sistema voltado à sala de aula, no qual o professor pode cadastrar suas turmas e suas próprias questões e acompanhar o desempenho dos alunos através de resultados estatísticos. Ambos permitem o armazenamento das provas na conta do usuário cadastrado e a possibilidade de imprimi-las. O Pratique OBI segue essa linha de recursos voltados a quem deseja selecionar questões e compor provas personalizadas, seja instrutor ou competidor. Um tal recurso, ao nosso conhecimento, não estava disponível para provas da OBI, motivando o desenvolvimento da ferramenta.

\section{Pratique OBI}

O Pratique OBI é um sistema web que permite a criação de provas personalizadas com questões da OBI na modalidade Iniciação, para serem utilizadas como simulados em treinos para a competição. A proposta é trazer um sistema de acesso fácil e intuitivo, com um banco de questões completo contendo todas as provas passadas e simplificando buscas por questões específicas. Vale destacar que, comumente, uma prova da OBI traz situaçõesproblema cujo enunciado se aplica a várias questões, mas também podem haver questões

\footnotetext{
${ }^{2}$ http://bebras.org/

${ }^{3}$ http://www.bebras.uk/bebrasapp.html

${ }^{4}$ https://digital.ftd.com.br/gestor.php

${ }^{5}$ https://www.gapi.com.br/
} 
isoladas, independentes de problemas.

Ao projetar o sistema, diferentes tipos de usuários foram definidos, cada um com suas permissões de acesso às funcionalidades. Cada usuário pode ter nenhuma ou ilimitadas permissões para a execução de certas funcionalidades, permitindo um sistema modular em questão de segurança e controle dos dados. Para isso, os usuários foram divididos em três grupos distintos de acordo com seu perfil:

- Usuário anônimo: Tem acesso à lista de provas anteriores e à busca por provas e problemas. Pode solicitar a criação de uma conta fornecendo informações básicas com confirmação de cadastro via e-mail.

- Usuário cadastrado: Deve realizar o registro no sistema. Tem acesso às mesmas funcionalidades que o usuário anônimo, mas também pode criar provas personalizadas. Tem acesso à página de provas criadas, no qual pode editar, excluir, visualizar e realizar download de suas provas.

- Administrador: Pode cadastrar provas completas da OBI e demais edições sobre os usuários e conteúdo cadastrados.

A Figura 1 apresenta a tela inicial do sistema, que pode ser acessado em: https://pratiqueobi.herokuapp.com. São oferecidos dois recursos principais: a criação de uma prova e a busca por provas, incluindo problemas e questões. Além disso, todos usuários têm acesso a uma página intitulada "Provas Passadas", contendo uma lista de todas as provas passadas da OBI com link para a prova oficial e link para a visualização da prova no sistema. O usuário cadastrado tem acesso à página "Minhas provas", onde ficam armazenadas todas as provas criadas, podendo editá-las, excluí-las ou visualizá-las para download.

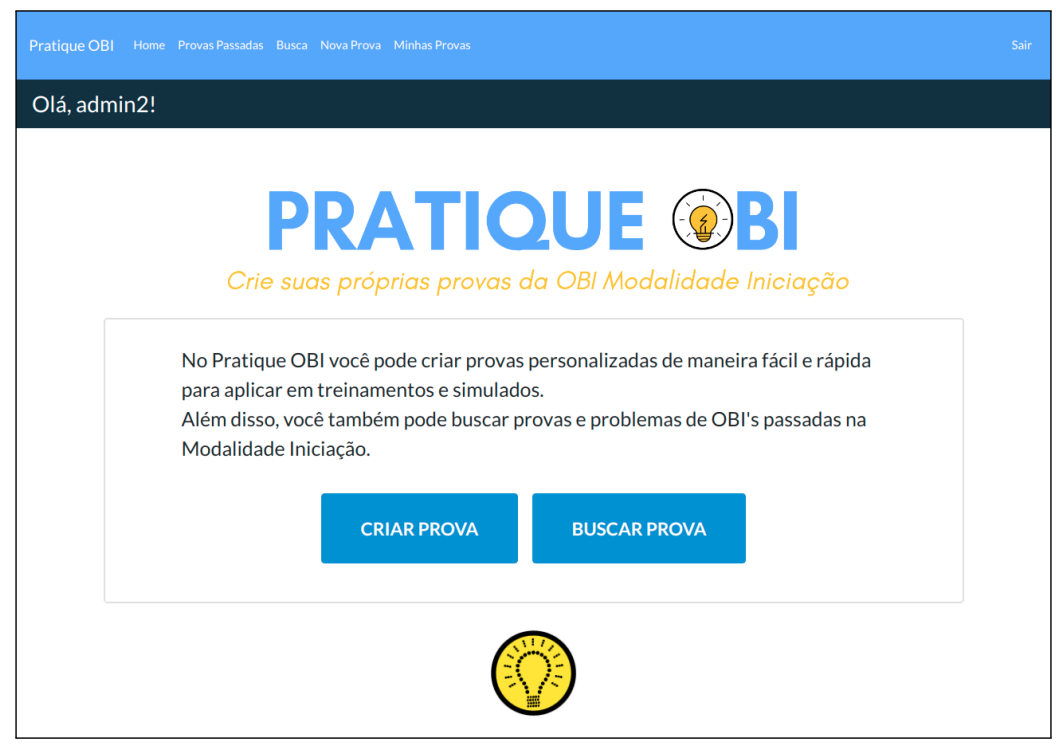

Figura 1. Página inicial do Pratique OBI

Todos usuários podem acessar a busca por provas ou por problemas, que se concentra em uma mesma página, uma abaixo da outra, visando facilitar o acesso. A Figura 2 apresenta a busca por provas específicas, classificadas por ano, nível e fase. Cada prova é associada a um link para o documento oficial da prova e um link para o gabarito, hospedados no site da OBI. 
A Figura 3 apresenta a busca por problemas. Cada problema da modalidade Iniciação foi previamente classificado seguindo a sugestão do livro "Jogos de Lógica", por tipos e por regras, não excludentes, sendo elas: questões de ordenação, de agrupamento, de grupos ordenados e de cálculo, contendo regras de posicionamento, de atribuição, de combinação, de sequenciamento e condicionais. Assim, os problemas foram catalogados por prova (ano, nível e fase), por suas classificações de tipos e regras, e pelos seus títulos. Cada problema é associado a um link para uma página contendo todas as questões do respectivo problema, incluindo imagens, alternativas e gabaritos.

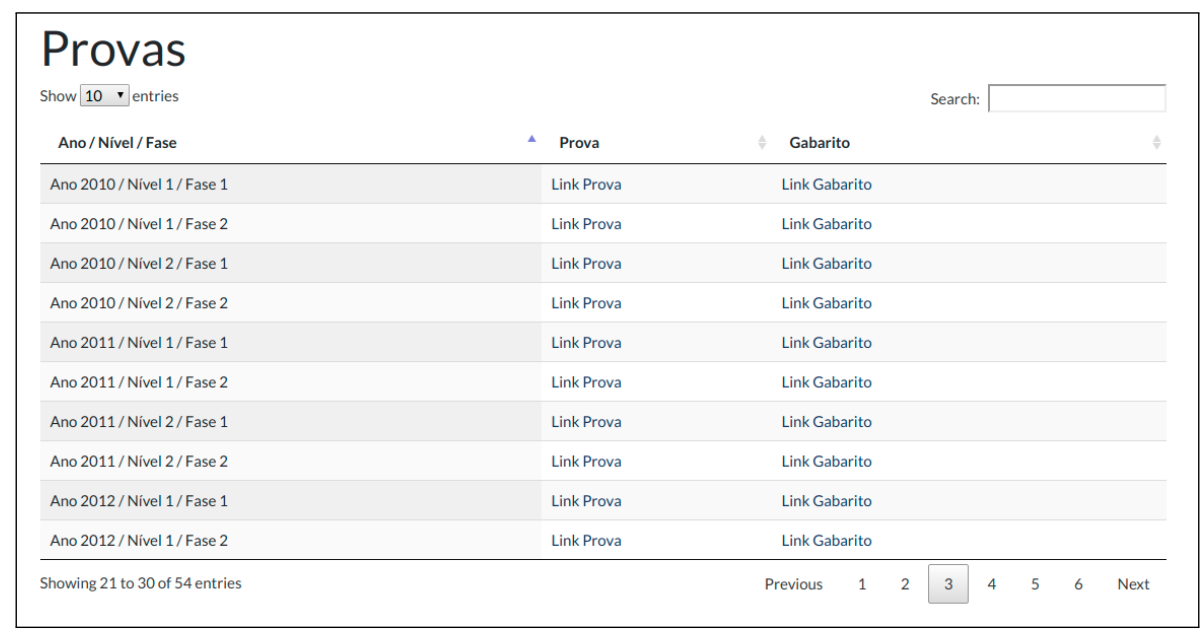

Figura 2. Busca por provas da OBI na modalidade Iniciação

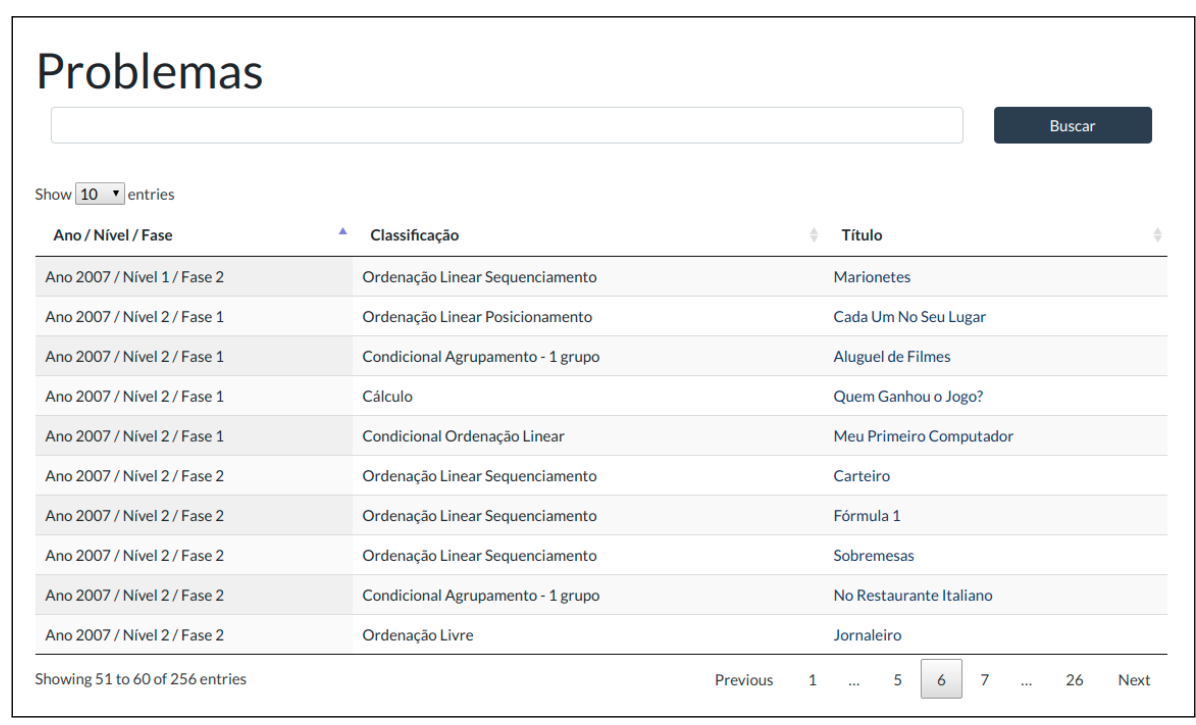

Figura 3. Busca por problemas da OBI na modalidade Iniciação

A Figura 4 apresenta a página de criação de uma prova personalizada. Nela, o usuário cadastrado define um "Título", um "Ano" para facilitar a identificação da prova nova em relação a outras já criadas, uma "Dificuldade" (muito fácil, fácil, médio, difícil e muito difícil) esperada para as questões que irão compor a prova e pode inserir "Observações" pertinentes. Apenas o campo de "Título" é obrigatório. No topo da página existe um breadcrumb, que serve como uma linha do tempo para localizar o usuário ao longo do processo de criação de uma prova. 
Na etapa de "Buscar Questões", o usuário cadastrado acessa uma busca semelhante à apresentada na Figura 3, na qual pode navegar por problemas cadastrados no banco de dados. A etapa seguinte ocorre após a seleção do problema desejado. São apresentadas todas as questões do problema e o usuário as escolhe clicando em uma caixa de seleção, podendo selecionar todas as questões de um mesmo problema ao apertar em um botão. Podem ser adicionadas questões de diferentes problemas, podendo-se retornar à etapa anterior. Na etapa final, é apresentada a prova pronta, com as opções de download da prova em PDF, em DOCX, ou armazenamento no Google Drive.

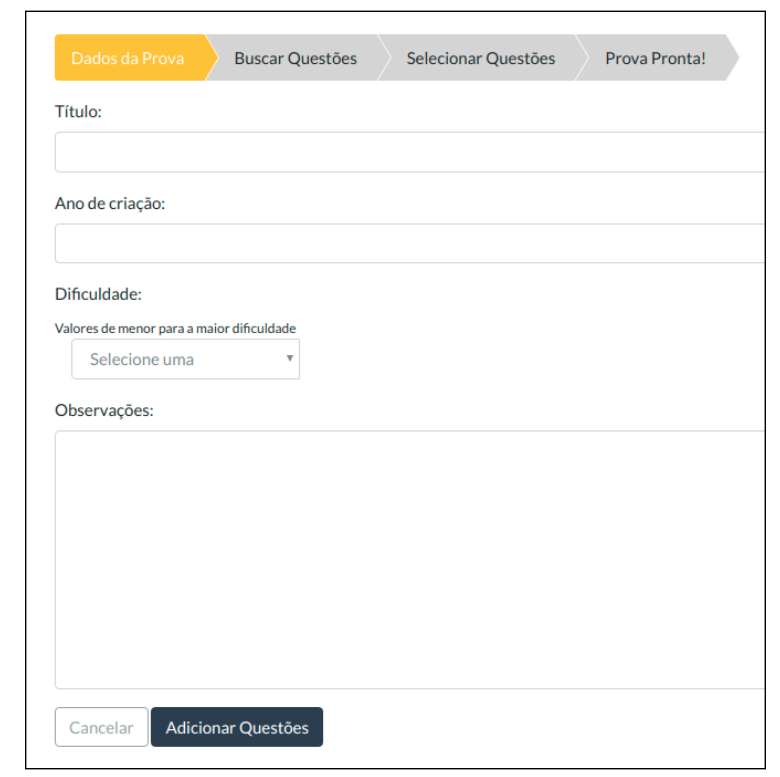

Figura 4. Página de criação de uma prova personalizada

O sistema foi hospedado na plataforma $\mathrm{Heroku}^{6}$, que disponibiliza um ambiente de computação em nuvem para a implantação de aplicações com suporte a diversas linguagens de programação e ferramentas. Algumas funcionalidades são disponibilizadas de forma paga e outras gratuitas mas com limitações. Uma das ferramentas disponibilizadas gratuitamente é o banco de dados chamado "Heroku PostGres", baseado no banco de dados PostgreSQL ${ }^{7}$, porém ele tem limite de tamanho da tabela do banco de dados, resultando em alterações no banco de dados inicialmente implementado com SQLite ${ }^{8}$.

Visando automatizar e simplificar o processo de criação de provas personalizadas, decidiu-se desenvolver o sistema com a linguagem Python e com o framework Django 9 . A maior vantagem do Django é que ele encoraja um desenvolvimento de design limpo, rápido e pragmático, oferecendo acesso a diversas funcionalidades que servem como facilitadores para a realização de processos específicos, o que acaba permitindo aos seus desenvolvedores focarem apenas na implementação do projeto.

O sistema de busca por provas e questões foi implementado com a biblioteca Django Watson ${ }^{10}$. Esta biblioteca permite realizar pesquisas utilizando apenas uma li-

\footnotetext{
${ }^{6}$ https://www.heroku.com

${ }^{7}$ https://www.postgresql.org

${ }^{8}$ https://www.sqlite.org/index.html

${ }^{9} \mathrm{https}: / / \mathrm{www}$. djangoproject.com

${ }^{10} \mathrm{https} / / /$ github.com/etianen/django-watson/wiki
} 
nha de código, dividindo a string de busca em palavras individuais e procurando cada palavra em campos relevantes em várias tabelas do banco de dados. Watson permite ordenar os resultados por relevância, além de ser rápido e escalável para a maioria dos casos de uso. Na ferramenta, a entrada do usuário é filtrada pelo Watson nos campos de títulos, enunciados e classificações dos problemas.

Para proporcionar o download das provas criadas, foram utilizadas ferramentas auxiliares, como a biblioteca Python Docx ${ }^{11}$, que permite a criação de documentos editáveis no formato DOCX, e a REST API do Google Drive ${ }^{12}$, que permite interação com a conta do Google Drive do usuário, podendo salvar e compartilhar documentos na plataforma, por exemplo.

A Figura 5 sumariza a parte principal do modelo de dados do projeto, com enfoque nas aplicações relacionadas às provas, ao usuário e ao registro. Cada prova tem vários problemas com classificações pré-definidas. Cada problema pode ter várias questões com uma alternativa para cada. O usuário deve realizar o registro para poder criar provas personalizadas, acessando e selecionando questões cadastradas.

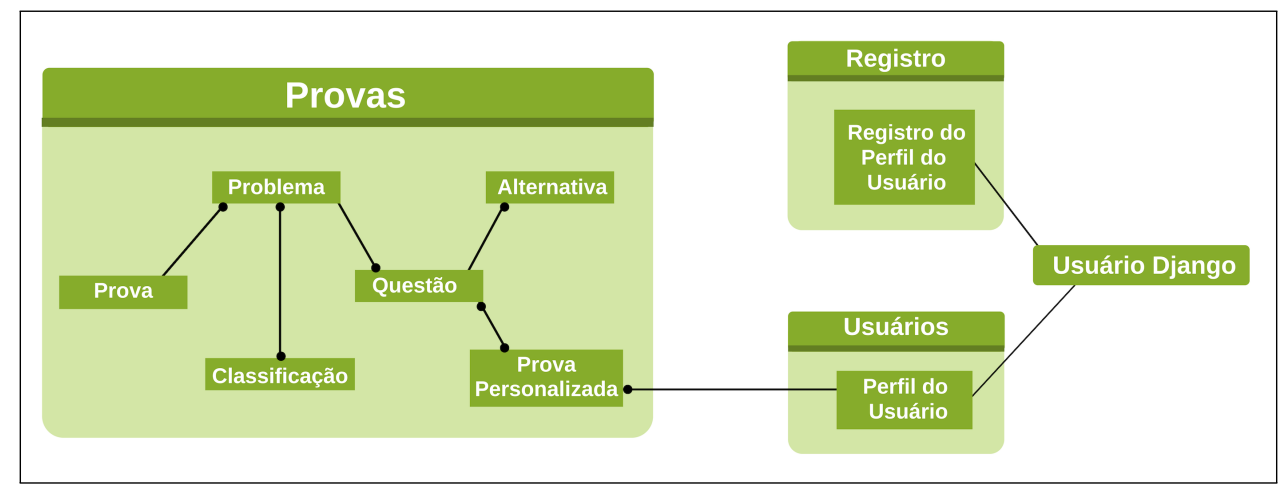

Figura 5. Subsistema de modelo de dados do projeto

\section{Testes e Resultados}

Após a implementação das principais funcionalidades do sistema (busca, criação da prova e download em PDF e DOCX), foram realizados testes com usuários. No momento dos testes, havia uma busca mais básica do que a apresentada anteriormente, com uma página para a busca por provas e outra página para a busca por problemas. Além disso, durante a criação da prova, o usuário acessava apenas a busca por provas para selecionar suas questões, o que era mais custoso do que acessar os problemas diretamente.

Os testes foram realizados com um total de 11 usuários. Todos eram graduandos em Ciência da Computação ou Sistemas de Informação da Universidade Federal de Santa Maria. Dentre eles, 6 eram instrutores colaboradores em um programa de extensão universitária que realiza treinos para a OBI há vários anos. Neste programa, em anos anteriores, colaboradores iniciaram a classificação das questões da OBI, que foi concluída para disponibilização no sistema Pratique OBI.

Foram disponibilizadas credenciais para login no sistema, facilitando o foco na criação de provas e navegação no site e não no cadastro, por exemplo. Os testes foram

\footnotetext{
${ }^{11}$ https://python-docx.readthedocs.io/en/latest

${ }^{12} \mathrm{https} / / /$ developers.google.com/drive/api/v3/about-sdk
} 
elaborados com tarefas que englobassem todas as funcionalidades existentes no sistema e que também demonstrassem se a descrição da página inicial estava compreensível para quem desconhecia a OBI. Foram criadas cinco tarefas no total:

1 Descubra qual é a primeira questão da prova de 2006 fase 1 e nível 2.

2 Verifique se existe algum problema com o nome "Excursão".

3 Verifique quantos problemas existem na prova de 2005 nível 2.

4 Crie uma prova com pelo menos 5 questões e faça download dela.

5 Mude o nome da prova criada anteriormente e adicione mais 2 questões à ela. Faça download desta prova alterada.

As tarefas foram repassadas aos testadores através de um documento no repositório do projeto, contendo descrição sobre o Pratique OBI, instruções sobre os testes e um link para o formulário avaliativo sobre o uso do sistema. No formulário, haviam questões sobre o ambiente de teste dos usuários e questões sobre cada tarefa, perguntando se o usuário havia conseguido as completar ou não e se encontrou dificuldades no percurso. Ao final, deixou-se um campo livre para críticas e sugestões.

Os resultados do formulário mostraram que, em geral, os usuários conseguiram realizar as tarefas satisfatoriamente. Durante os testes, alguns usuários se depararam com falhas específicas como: (i) não conseguir realizar o download de provas com imagens no formato PDF, que ocorreu devido à inserção incorreta do caminho do arquivo de imagem no código fonte do sistema, (ii) buscas livres por provas existentes retornando resultados vazios, o que era uma falha esperada pois o banco de dados com todas provas ainda não estava completo, e (iii) erro ao renomear uma prova, pois a implementação não estava reconhecendo provas com "Dificuldade" não selecionada.

Outros participantes sugeriram melhorias na interação com o usuário, pois consideraram difícil encontrar alguns botões e utilizar a busca, relatada como "não intuitiva". A seguir apresenta-se alguns relatos de usuários:

"Quando abre a página de visualização da prova seria interessante aparecer os botão ( sic) de "editar" e "excluir"."

"Dá para excluir questões pontuais de uma determinada prova?"

"Esse é um espaço para "críticas e sugestões" mas deixo aqui minha parabenização pelo trabalho, está muito bom!”

"Gostei muito dos diferentes métodos de pesquisa, acho que seria bom, inclusive, uma caixa de pesquisa na tela inicial. Tive dificuldades na hora de criar a prova (não consegui), seria interessante também que na hora de criar a prova, houvesse a pesquisa por problemas, não somente por provas."

Após os testes, foram corrigidas as falhas de implementação, melhorou-se a busca geral e a busca para criação da prova, que permaneceram conforme apresentadas nas Figuras 2 e 3, e alguns botões e links no visual do site para melhor interação. Também foram adicionados o recurso de editar e excluir provas criadas, a confirmação do cadastro via e-mail, a possibilidade de salvar a prova no Google Drive e alterou-se o banco de dados do SQLite para o PostGres, visto o maior suporte para hospedagem no Heroku. Outras sugestões como a possibilidade de excluir questões específicas de uma prova e aprimorar o sistema para se tornar responsivo em dispositivos móveis estão sendo estudadas. 


\section{Conclusão e Trabalhos Futuros}

Neste trabalho, apresentou-se o Pratique OBI, um sistema web que proporciona acesso facilitado a todas questões da OBI na modalidade Iniciação, e permite a criação de provas personalizadas para fins de estudo. O sistema pode ser acessado com todas funcionalidades disponíveis gratuitamente em: https://pratiqueobi.herokuapp.com.

O Pratique OBI é focado em oferecer um ambiente com todos problemas de lógica da OBI, na modalidade Iniciação, cadastrados e classificados, juntamente com opções de filtragem para que o usuário consiga facilmente consultar provas ou problemas. Com esses recursos, é notória uma grande diversidade de opções para o usuário criar uma prova personalizada, permitindo ampliar suas práticas de resolução ao se preparar para uma edição da OBI.

Como trabalhos futuros, planeja-se oferecer a possibilidade de gerar documentos semelhantes às provas oficiais da OBI, de modo a preparar melhor os participantes em treinos com simulados. Para isso, está sendo implementada a opção de criar provas com imagens em alta qualidade de questões extraídas de provas oficiais. Também, pretende-se disponibilizar um gabarito correspondente a cada prova, semelhante ao usado na OBI.

\section{Agradecimentos}

Os autores deste trabalho agradecem aos colaboradores do programa de extensão universitária "Clube de Computação da UFSM", que oferece treinos para a OBI utilizando questões de provas passadas, o que inspirou a criação do Pratique OBI. Também aos alunos de graduação em Ciência da Computação, Otávio Oliveira Deon e Marlon Leoner, que colaboraram com a classificação e coleta de questões e provas passadas da OBI em diferentes formatos, facilitando a inserção no sistema.

\section{Referências}

Dagienè, V. (2006). Information technology contests - introduction to computer science in an attractive way. Informatics in Education, 5(1):37 - 46.

Dagienè, V. and Futschek, G. (2008). Bebras international contest on informatics and computer literacy: Criteria for good tasks. In Mittermeir, R. T. and Sysło, M. M., editors, Informatics Education - Supporting Computational Thinking, pages 19-30, Berlin, Heidelberg. Springer Berlin Heidelberg.

Dantas, V., Nogueira, A., Alisson, N., Raniery, D., Raul, J., Soares, R., Sampaio, R., Kelson, W., and Costa, T. (2013). Uma metodologia para estimular o raciocínio lógico baseada na reflexão crítica e no uso de jogos digitais. In Anais do Workshop em Informática na Escola - (WIE), pages 353 - 361.

de Almeida Machado, E. Z., Vasconcelos, I. R., Amorim, K. M., Andrade, A. M. S., Barreto, L. P., and Santos, D. A. (2010). Uma experiência em escolas de ensino médio e fundamental para a descoberta de jovens talentos em computação. In Anais do Workshop sobre Educação em Computação - (WEI), pages 798 - 807. Sociedade Brasileira de Computação.

de Barros, L. G. and Oeiras, J. Y. Y. (2010). Extensão universitária em computação por meio da Olimpíada Brasileira de Informática. In Anais do Workshop sobre Educação em Computação - (WEI), pages 788 - 797. Sociedade Brasileira de Computação. 
de Barros, L. G., Ribeiro, S. P. S., and Oeiras, J. Y. Y. (2009). Projeto de extensão universitária para apoio e realização da Olimpíada Brasileira de Informática em escolas. In Anais do Workshop sobre Educação em Computação - (WEI), pages 567 - 576.

de Oliveira Anido, R. and Menderico, R. M. (2007). Brazilian Olympiad in Informatics. Olympiads in Informatics, 1:5 - 14.

dos Santos, E. R. S., Oliveira, F. C. S. O., and Neto, I. B. M. (2015). Raciocínio lógico e computação: Descobrindo estratégias de ensino por meio da Olimpíada Brasileira de Informática. In Anais do Workshop em Informática na Escola - (WIE), pages 266 270 .

Martins, W. S. (2011). Jogos de Lógica: Divirta-se e prepare-se para a Olimpíada Brasileira de Informática. Editora Vieira.

Piaget, J. and Inhelder, B. (1975). Gênese das estruturas lógicas elementares. Editora Zahar.

Trindade, R. G., da Silveira Schneider, C. A. D., and Charão, A. S. (2017). Logic in a Logic Way: um aplicativo para exercitar a resolução de problemas de lógica da Olimpíada Brasileira de Informática. In Anais do Workshop sobre Educação em Computação (WEI), pages $2237-2246$. 